\title{
Treatment of Pregnancy-Associated Breast Cancer
}

\author{
BERNADETTE LABRIOLA, RN, MSN, FNP-C
}

From Duke Cancer Institute, Duke University Health Systems, Durham, North Carolina

Author's disclosure of conflicts of interest are found at the end of this article.

Correspondence to: Bernadette Labriola, RN, MSN, FNP-C, 10 Bryan Searle Drive, Durham, NC 27710. E-mail: bernadette.labriola@duke.edu https://doi.org/10.6004/jadpro.2019.10.7.5 (c) 2019 Harborside $^{\mathrm{TM}}$

\section{CASE STUDY}

$\mathrm{KS}$ is a 33-year-old Caucasian married woman who works full time as an accountant and has one daughter who is 2 years old. She enjoys reading and exercising in her spare time. She initially presented in July 2015 at the age of 31 years with a 1-cm breast mass in the left inner breast, which prompted a mammogram to be obtained. The mammogram revealed diffuse and occasionally grouped left breast calcifications. Additionally, there was focal edema at the site of the mass.

A follow-up mammogram was recommended to document stability in 6 months, which demonstrated an interval increase in number and size of segmental pleomorphic calcifications in the lower inner breast spanning $6 \mathrm{~cm}$ in size. A stereotactic core needle biopsy was completed and revealed high-grade ductal carcinoma in situ (DCIS) that was estrogen receptor (ER) and progesterone receptor (PR) positive.

\section{Surgical Treatment}

KS underwent genetic testing due to her young age at diagnosis of noninvasive breast cancer. She was tested with the breast/ovarian cancer panel, which was negative for mutation.

She preceded to bilateral nipple-sparing mastectomy with left sentinel node biopsy and immediate implant reconstruction in February 2016. The operative pathology revealed $3.3 \mathrm{~cm}$ of high-grade DCIS. The surgical margins were negative (less than $1 \mathrm{~mm}$ posteriorly and less than $2 \mathrm{~mm}$ anteriorly). There was 1 sentinel node and 2 nonsentinel nodes negative for malignancy. The right breast was negative for cancer and both retro areolar margins were negative.

KS did well during the ensuing routine follow-up every 6 to 12 months in surgical oncology at an academic medical center. She was not recommended to take adjuvant endocrine therapy given the benefit of bilateral mastectomy.

In 2017, at a routine oncology follow-up visit, she expressed a desire to have more children. After a negative clinical exam, she and her husband were advised that future contraception may be pursued.

\section{Pregnancy-Associated Breast Cancer Diagnosis}

KS was in her usual state of good health when she noticed a left breast mass in the inferior reconstructed breast in June 2017. She presented 
to the nurse practitioner (NP) in the surgical oncology clinic for evaluation. At that time, she was 17 weeks pregnant and being seen in the same facility for high-risk obstetrics and gynecology care. KS had no other concerning symptoms for recurrent cancer, and her pregnancy had been progressing smoothly.

The surgical oncology NP noted a 1-cm firm superficial mass in the breast at 6 o'clock. She had no other bilateral breast findings or adenopathy. Upon review of systems, she denied new persistent headache, shortness of breath, abdominal pain, weight loss, night sweats, or fatigue.

Due to her pregnancy and the superficial presentation of the breast mass, a left breast ultrasound was ordered. It revealed an $8-\mathrm{mm}$ irregular hypoechoic mass $7 \mathrm{~cm}$ from the nipple at the 6 o'clock position in the reconstructed breast.

A diagnostic workup ensued with a left breast ultrasound-guided core needle biopsy. KS was given a diagnosis of clinical stage T1b, NO, grade 2 invasive micropapillary carcinoma: ER positive (Allred 6), PR positive (Allred 8), HER2/neu, immunohistochemistry 3+, and fluorescence in situ hybridization amplified.

After discussion of this recurrent cancer diagnosis, her team opted for a bilateral diagnostic mammogram (with abdominal shielding) and bilateral axillary and breast ultrasound to evaluate the contralateral breast and lymph nodes. There was no adenopathy, a small amount of accessory breast tissue in the right axillary tail region, and a biopsy clip was noted in the left inferior breast at 6 o'clock. The new cancer was not seen on mammogram, likely due to the proximity to the implant.

reast cancer is the second most common cancer diagnosis during pregnancy (Shachar et al., 2017). It occurs in approximately $0.2 \%$ to $2.6 \%$ of all woman diagnosed with cancer (Shachar et al., 2017). Cervical cancer during pregnancy is the most common cancer diagnosis during pregnancy (Shachar et al., 2017). Pregnancyassociated breast cancer (PABC) is defined as occurring during pregnancy, lactation, or within 1 year after delivery (Toesca, Gentilini, Peccatori, Azim, \& Amant, 2014). Pregnancy-associated breast cancer occurs in 1 of every 3,000 to 10,000 pregnancies (Shlensky, Hallmeyer, Juarez, \& Parilla, 2017).

The patient with PABC is between 23 and 47 years of age, with a median age of 33 years (Brooks et al., 2012; Rovera et al., 2013). A diagnosis during pregnancy remains rare in the population but may increase as women are conceiving later in their mid-30s to 40s (Rovera et al., 2013; Toesca et al., 2014). Women with breast cancer before age 40 are more likely to have PABC. $B R C A 1$ and 2 carriers have a higher risk for PABC.

Pregnancy increases the risk of breast cancer initially after pregnancy; that effect lasts for approximately 10 to 15 years, then imparts a protective effect afterwards. This may be related to the rapid growth of breast cells during pregnancy. If there is any genetic damage in the breast cell, it is copied as the cells grow. The chance of having genetic damage to cells increases with age (Johnson, Anders, Litton, Ruddy, \& Bleyer, 2018).

For women who are older at a first pregnancy, there is a longer duration of increased risk before the protective effect of pregnancy occurs. In a Norwegian study, women who conceived over age 35 had a permanent increased risk of breast cancer compared to nulliparous women (Yu, Cheung, Leung, Leung, \& Kwan, 2017). Among BRCA carriers, the high levels of circulating estrogen may accelerate the malignant changes that have already begun related to having a mutation status (Rovera et al., 2013).

\section{DIAGNOSTIC DELAY}

Core needle biopsy is the gold standard in which histologic grade, receptor status, and human epidermal growth factor receptor 2 (HER2) status are used to establish a diagnosis of an abnormal breast finding (Yu et al., 2017). The majority of PABC diagnoses are ductal, estrogen receptor-negative, high-grade tumors with lymphovascular invasion noted (Rovera et al., 2013).

KS did not experience a delay in a diagnosis of $\mathrm{PABC}$ as she was receiving close follow-up for a high-risk pregnancy and routine oncology followup. Additionally, the breast mass was superficial and palpable, which allowed for quick detection on self-exam and clinical exam. 
There may be some delays in securing a diagnosis of PABC due to normal changes in breast density during pregnancy. Despite these breast changes, a mammogram has a sensitivity of more than $80 \%$ during pregnancy (Botha, Rajaram, \& Karunaratne, 2018; Cordeiro \& Gemignani, 2017; Johansson, Weibull, Fredriksson, \& Lambe, 2019; Rovera et al., 2013; Toesca et al., 2014). Changes in the breast tissue may be the reason for PABC to present with a higher grade and larger tumor size. The median tumor size is $3.5 \mathrm{~cm}$, and these cases are more likely to display lymphovascular invasion (Botha et al., 2018; Toesca et al., 2014). Placenta metastasis is rare (Botha et al., 2018; Yu et al., 2017). These delays may affect future prognosis (Johansson, Weibull, Fredriksson, \& Lambe, 2019; Rovera et al., 2013).

Epidemiologic data indicate that a breast cancer diagnosis during lactation exhibits the most aggressive biological environment and an increase in cause-specific death. Women who are diagnosed with breast cancer within 1 year of delivery have a shorter time to relapse and an increased risk of metastatic death. Lactating stromal adipose cells express higher levels of inflammatory cytokines that are highly angiogenic and growth-promoting, leading to more aggressive tumors (Yu et al., 2017).

\section{DIAGNOSIS OF PABC}

Approximately $80 \%$ of the women presenting to clinic with a mass will have a benign finding. Breast masses lasting longer than 2 to 4 weeks and associated with skin changes raise concern for a suspicious abnormal mass. Both mammogram and ultrasound imaging modalities are used in pregnant women to assist in the diagnosis of a suspicious breast mass. Radiation exposure to the fetus is minimal with a mammogram and may be done with an exposure of only $0.004 \mathrm{~Gy}$, which is below the threshold for a detrimental effect (Rovera et al., 2013; Yu et al., 2017). The International Commission on Radiological Protection notes that there is no detrimental effect of significance at a threshold dose of $100 \mathrm{~Gy}$ (Yu et al., 2017). Shielding of the abdomen with a lead apron will decrease fetal exposure to radiation scatter (Botha et al., 2018).

The use of breast MRI in pregnancy is not recommended given the difficulty distinguishing malignant from physiologic hypervascularization that occurs during pregnancy. There is no safety data on the commonly used contrast gadolinium in pregnancy (Cordeiro \& Gemignani, 2017). Fetal abnormalities have been noted in exposed rats (Rovera et al., 2013; Yu et al., 2017).

In KS's case, the oncology NP was able to expedite the scheduling of a multidisciplinary team meeting at the request of her obstetrics/gynecology provider. The obstetrics/gynecology provider made note that if KS were to consider pregnancy termination, the window for decision-making was within a 1-to-2-week timeframe.

Unless there is a strong clinical suspicion for metastatic disease, a staging workup is not routinely indicated (National Comprehensive Cancer Network [NCCN], 2018; Yu et al., 2017). If a staging workup is indicated, this may include an ultrasound of the abdomen/liver or a noncontrast MRI of the abdomen for evaluation of liver or bone metastases. Computed tomography of the abdomen is avoided due to dangerous levels of radiation (NCCN, 2018; Rovera et al., 2013). KS did not have concerning symptoms for metastatic recurrence and did not require staging scans.

\section{SURGICAL MANAGEMENT OF PABC}

Surgery for breast cancer is recommended and considered safe in all trimesters of pregnancy (Table 1). Surgery performed after 12 weeks confers the lowest risk of miscarriage, similar to the risk of spontaneous miscarriage in the first trimester of a routine pregnancy (Basta, Bak, \& Roszkowski, 2015; Rovera et al., 2013; Yu et al., 2017). Women may have the option of breast-conserving surgery based on tumor size, characteristics, and stage at time of presentation. A woman's preferences are taken into account when making surgical choices during shared decision-making. Surgical complications may include an increased risk of low birth weight due to premature labor or intrauterine growth retardation (Rovera et al., 2013).

An invasive cancer diagnosis will warrant lymph node evaluation with either sentinel node biopsy or axillary lymph node dissection as indicated, which can be safely carried out at experienced breast cancer treatment centers (Rovera et al., 2013). Axillary lymph node evaluation with sentinel node biopsy appears to be safe during 
pregnancy with an injection of radiocolloid technetium to identify the sentinel lymph node. A small injection of radioisotope is injected into the periareolar breast (Toesca et al., 2014). This will drain to the sentinel node, which may be the first possible lymph node basin affected with lymph node metastasis. Blue dye is not used in pregnant women due to the unknown potential risk for teratogenicity. In some cases anaphylaxis has been reported; additionally, adverse outcomes of intestinal atresia and fetal demise may occur (Cordeiro \& Gemignani, 2017; Yu et al., 2017).

In a 2015 study by Loibl and colleagues, it was noted that the absorbed radiation dose from technetium from sentinel node injection over the abdomen at the level of the fetus measured by dosimeter was less than $10 \mathrm{~Gy}$. A dose of $20 \mathrm{~Gy}$ is comparable to 1 to 2 days of natural background radiation. The risk of leukemia and cancer is $1.4 \%$ with a dose of $10 \mathrm{~Gy}$ (Cordeiro \& Gemignani, 2017; Toesca et al., 2014; Yu et al., 2017).

The sentinel node procedure can be done the day of surgery to further reduce the risk of radiation exposure to the fetus. Typically one to three lymph nodes will be localized by colloid injection and removed for evaluation of lymph node metastases (Shachar et al., 2017).

Axillary staging with nodal evaluation with sentinel node biopsy or lymph node dissection provide important prognostic information and allows for better local control of breast cancer. For a woman undergoing a sentinel node biopsy, the risk of lymphedema is $5.3 \%$ compared to $11.8 \%$ with an axillary lymph node dissection ( $\mathrm{Yu}$ et al., 2017).

Immediate breast reconstruction after a mastectomy may be offered in some cases for in situ carcinoma or for early-stage breast cancer, with sentinel node status being evaluated prior to the definitive breast surgical procedure (Cordeiro \& Gemignani, 2017). At the time of mastectomy, a tissue expander may be placed, which adds minimal time to the surgical case and time under anesthesia. The tissue expander would be exchanged for an implant after delivery. Immediate breast reconstruction with autologous tissue would not be considered due to the long operative times, blood loss, and possible postoperative complications (Toesca et al., 2014).

\begin{tabular}{|ll|}
\hline Table 1. Timing of PABC & Treatment \\
\hline Surgery & 2nd-3rd trimester \\
Chemotherapy & 2nd-3rd trimester \\
Radiation & Post delivery \\
\hline
\end{tabular}

\section{CASE STUDY CONTINUED}

KS and her husband were understandably upset about this diagnosis of recurrent invasive breast cancer during pregnancy. After becoming well informed by team members, KS elected to undergo treatment of PABC.

KS was anxious and tearful during her oncology consult. She was offered supportive counseling with an oncology counselor to support her in her treatment decisions. Additionally, she was well supported by her husband, family, and friends. She declined referral for children's counseling for her preschool-aged daughter.

KS agreed to move forward with excision of the left breast mass and an attempt at sentinel node biopsy under general anesthesia or regional anesthesia depending on Obstetrics' input. Fetal heart monitoring was performed by the Obstetrics team both before and after surgery. The anatomy ultrasound was moved up prior to surgery to verify fetal health and growth. Staging studies were again deferred and would be based on surgical operative pathology results. Fetal growth was normal and on target for the second trimester of pregnancy.

KS was recommended to have chemotherapy in the adjuvant setting to begin 2 to 3 weeks after postoperative recovery. She recovered uneventfully from surgery with minimal pain. At the l-week return postoperative visit, the left breast lumpectomy and axillary incisions were healing well, with excellent cosmetics.

$\mathrm{KS}$ was recommended to have doxorubicin and cyclophosphamide given every 3 weeks for 4 cycles over a 12 -week period during the second trimester of pregnancy. Routine interval ultrasound was done to evaluate fetal growth before treatment and every 3 to 4 weeks prior to chemotherapy. Other forms of fetal assessment may be of value: In some cases, umbilical artery Doppler ultrasound to assess placenta status, Doppler study of the fetal middle cerebral artery to exclude fetal anemia, and serial fetal echocardiogram when using anthracycline drugs that can be cardiotoxic 
(Framarino-Dei-Malatesta, Sammartino, \& Napoli, 2017). Assessment of amniotic fluid volume is also necessary because it can decrease reversibly with the use of some drugs (Yu et al., 2017).

An implantable port was placed under local anesthesia, and chemotherapy began 2 weeks after breast surgery, from July to September 2017. KS was to finish chemotherapy by 32 to 35 weeks and was scheduled for a Caesarean section based on the treatment timing. KS had a second planned Caesarian section (37 weeks 1 day) under spinal anesthesia in November 2017. She had a male baby weighing 3,110 g with an Apgar score of 8/9. There were no complications. She had a bilateral riskreducing salpingectomy with negative pathology. The baby and mother did well post partum with bottle feeding planned. After delivery, KS was placed on a 6-week course of prophylactic enoxaparin sodium (Lovenox) in the setting of postpartum breast cancer and being at a higher risk for blood clots.

Upon delivery, adjuvant chemotherapy with a weekly taxane (paclitaxel) and trastuzumab (Herceptin) was given for 12 weeks. She completed 1 full year of trastuzumab post delivery, which ended in November 2018. She was to receive breast radiation after the completion of taxanebased chemotherapy.

KS was well supported by her mother and husband. Her daughter did not have any behavioral issues or difficulty coping with her family changes related to her mother's treatment and continued to attend daycare. KS worked full time during treatment with intermittent time off for chemotherapy and recovery days. KS had a prior history of depression and anxiety and was seeing a psychiatrist regularly who managed medications, and she continued to be stable on sertraline (Zoloft).

\section{CHEMOTHERAPY TREATMENT}

Pregnant patients with a diagnosis of breast cancer should be offered the same breast cancer treatment as nonpregnant patients (Basta et al., 2015). Pregnancy termination may be considered during treatment planning; however, it has not been shown to improve outcomes. Women with an advanced-stage breast cancer or with high-grade or aggressive primary tumors with a diagnosis in the first trimester may elect pregnancy termination to allow them to begin chemotherapy sooner (Yu et al., 2017).

For PABC patients, chemotherapy-induced reproductive toxicity may cause permanent amenorrhea, menstrual irregularity, and subfertility, but this depends on the dose, agent, and patient age (Yu et al., 2017). Patients may consider a referral to a reproductive fertility specialist if future childbearing is desired (Knabben \& Mueller, 2017; Trivers et al., 2014).

Fertility preservation may occur with embryo or oocyte cryopreservation to protect future fertility. Ovarian stimulation may be carried out before beginning chemotherapy but may result in a delay in treatment and increased estradiol levels (Tomasi-Cont, Lambertini, Hulsbosch, Peccatori, \& Amant, 2014; Garrido-Marín, 2019; Yu et al., 2017). General chemotherapy risks include preterm delivery, low birth weight, transient tachypnea of newborn, and transient neonatal leukopenia. The reported fetal malformation risk following chemotherapy during the second and third trimesters is approximately $3 \%$, which is no higher than that for the general population (Black, Nichols, Eng, \& Rowley, 2017; Rovera et al., 2013; Shachar et al., 2017).

Most children show normal neurologic development after exposure to chemotherapy in utero, although behavioral, emotional issues, and risk for future malignancy need additional investigation for delayed complications (Danet et al., 2018; Gajjar, Martin-Hirsch, \& Martin, 2012).

The German Breast Group reported a multicenter study that included 197 patients who received chemotherapy during pregnancy (447 patients over 8 years). Overall, preterm delivery was noted in $50 \%$ as compared to $10 \%$ to $15 \%$ of the general population. Early delivery before 37 weeks is associated with a higher chance of side effects, malformations, or newborn complications. The proportion of malformations in the study was no different than that for the general population (approximately 9\%). In utero exposure to chemotherapy was related to lower birth weight and more complications, but these were not statistically significant and were likely related to premature delivery (Black et al., 2017; Yu et al., 2017)

Anthracycline-based regimens are the most widely used and have a favorable side-effect pro- 
file when administered during pregnancy. Assessment of maternal cardiac function is recommended by echocardiogram. Studies have not shown fetal cardiotoxicity in utero (Framarino-Dei-Malatesta et al., 2017). Common side effects for patients may include neutropenia, oral ulcers, anaphylaxis, constipation, tachycardia, and cellulitis.

Taxanes appear to be another alternative in the third trimester but have not been extremely well studied (Rovera et al., 2013). A meta-analysis showed that the addition of taxanes to anthracycline-based regimens resulted in a statistically significant reduction in the risk of relapse (relative reduction, $17 \%$ ) and death (relative reduction, $15 \%$ ). A disease-free survival benefit was independent of estrogen-receptor expression, degree of nodal involvement, and type of taxane used. Taxanes are substrates of the P-glycoprotein that is highly expressed on the maternal compartment of the placenta. P-glycoprotein protects the fetus against xenobiotics and might therefore reduce trans placental transfer of taxanes (Lambertini, Kamal, Peccatori, Del Mastro, \& Azim, 2015; Yu et al., 2017; Zagouri et al., 2013). Taxanes are metabolized by cytochrome $\mathrm{P} 450$ that increases by $50 \%$ to $100 \%$ during the third trimester, which may result in a shorter half-life and a reduced toxicity profile.

A dose-dense chemotherapy regimen is a hotly debated topic. In this fashion, chemotherapy would be given every 1 to 2 weeks instead of every 3 weeks (Cordeiro \& Gemignani, 2017). Dosedense chemotherapy may allow earlier completion before delivery, closer pregnancy monitoring, and a better toxicity profile. Granulocyte-colony stimulating factors may be recommended to limit the potential for neutropenia in the mother and have been safely administered in pregnant patients (Cordeiro \& Gemignani, 2017; Loibl et al., 2015).

Chemotherapy should be held 3 weeks before delivery or after 35 weeks of gestation to minimize the risk of sepsis and hemorrhage in the mother and the newborn (Rovera et al., 2013). Breast feeding can resume 3 to 4 weeks after the last administered dose of chemotherapy (Pistilli et al., 2013).

MONOCLONAL ANTIBODY THERAPY

Once a diagnosis of breast cancer is made, histology and cancer biomarkers are run on the core nee- dle biopsy specimen. HER2/neu status is reported as either positive or negative. A positive HER2/ neu status warrants treatment with targeted therapy with trastuzumab. This drug is given intravenously every 3 weeks after delivery for 1 year of therapy. Trastuzumab can affect cardiac function in the patient; therefore, a baseline and periodic diagnostic echocardiogram is performed to check cardiac ejection fraction. The dose may be held for reduced cardiac function and given once there is a return to normal baseline.

Meta-analysis with trastuzumab revealed the main adverse event to be oligohydramnios (61.1\%), with the incidence increasing with duration of treatment. Alteration is likely due to the effect on the fetal kidney, where the HER2 receptor is highly expressed. There is a risk for fetal renal failure with trastuzumab (Shlensky et al., 2017). Breastfeeding is contraindicated due to transmission of the drug in breast milk. The drug is not associated with impaired fertility (Yu et al., 2017)

\section{CASE STUDY CONTINUED}

KS was prescribed oral dexamethasone on days 1 to 4 of treatment and oral ondansetron for nausea on days 1 to 2 and then as needed. She experienced minor tingling in her fingers and toes that resolved after chemotherapy completion. After the first cycle of doxorubicin and cyclophosphamide, she experienced a prolonged headache for 1 to 2 days due to dehydration. This was treated with outpatient IV fluid replacement and did not occur on subsequent cycles.

Chemotherapy may cause nausea. Regimens generally include pre- and posttreatment medication with antiemetics. Serotonin antagonist and dexamethasone are the preferred antiemetics. Granulocyte-colony stimulating factors such as filgrastim (Neupogen) may be recommended to minimize potential maternal and fetal problems associated with neutropenia, and erythropoietin has been safely administered in pregnant patients (Yu et al., 2017).

Approximately 2 weeks after the completion of chemotherapy, KS moved forward to initial treatment planning for adjuvant radiation therapy in February 2018. Additionally, she began goserelin for ovarian suppression given by intramuscular injection every 3 weeks timed with trastuzumab 
doses. After the completion of trastuzumab, she received goserelin every 4 weeks.

She was instructed in the use of the breath hold technique during radiation treatments for protection of cardiac tissues. A pregnancy test was ordered and returned negative. The radiation plan targeted the reconstructed left breast as well as surrounding regional lymph nodes. Comprehensive nodal radiation treatment was elected. Morbidity was thought to be very low in this thin patient with no prior axillary dissection. A dose of 50 Gy in 25 fractions was planned and delivered over a 4-week period beginning in March 2018.

On follow-up clinical exams, KS tolerated radiation without fibrosis of the skin or capsular contracture of the left breast implant. Her newborn son was healthy and meeting expected milestones. Her family was thriving and had adjusted well to their new addition. She returned to work as an accountant full time after maternity leave.

\section{RADIATION TREATMENT}

Radiotherapy is contraindicated until after delivery unless it is used for life-saving issues or to preserve organ function (e.g., spinal cord compression in the mother). Exposure to radiation in utero can result in fetal malformations during this time of organogenesis, cause mental retardation, and may increase the risk of childhood cancers (Toesca et al., 2014; Rovera et al., 2013). Risks of radiotherapy may result in miscarriage and fetal growth restriction (Basta et al., 2015). If radiotherapy is indicated during pregnancy, fetal shielding of the pelvis should be considered; in some cases, elective early delivery may be an option. Fetal shielding can decrease the radiation dose by $50 \%$ to $75 \%$ (Botha et al., 2018; Toesca et al., 2014).

Substituting whole-breast therapy for partialbreast irradiation may be another alternative. Standard doses for external beam radiation to the breast range from 45 to 60 Gy and may result in fetal harm. However, the risk of local recurrence in young pregnant women is higher with the lower dose of radiation typically recommended in women over 60 years. Another option for radiation therapy delivery may be intraoperative electron beam radiotherapy with a single dose of radiation of $21 \mathrm{~Gy}$, which would reduce fetal exposure but may affect recurrence rates (Toesca et al., 2014).
Adjuvant radiotherapy is not an urgent procedure and is given in a delayed fashion after delivery (Toesca et al., 2014). Delaying treatment more than 12 weeks after delivery can increase the likelihood of axillary metastases. In a meta-analysis of 20 studies, a waiting time for radiation of 1 month is equivalent to a local recurrence risk of $1.0 \%$ per month of delay in starting treatment (Toesca et al., 2014). However, it is important to note that nonpregnant patients receiving neoadjuvant chemotherapy will also experience a delay of up to 6 months from surgery until radiation is given (Toesca et al., 2014; Yu et al., 2017).

\section{ENDOCRINE THERAPY}

Endocrine therapy for estrogen receptor-positive and progesterone receptor-positive breast cancer would be delayed until after delivery and avoided during breast feeding. Tamoxifen given during pregnancy and/or breast feeding can cause birth defects, including craniofacial malformations, ambiguous genitalia, and fetal death (Cordeiro \& Gemignani, 2017; Rovera et al., 2013; Shlensky et al., 2017). Tamoxifen is contraindicated when breast feeding due to transfer in breast milk. Long-term effects of the drug on female offspring are unknown.

\section{CASE STUDY CONTINUED}

KS requested a consultation in the multidisciplinary sexual health clinic for complaints of dyspareunia and vaginal dryness caused by letrozole. Letrozole is used to reduce estrogen in hormonepositive breast cancer. She met with a pharmacist to discuss nonhormone-based treatments for vaginal dryness, physical therapy to determine other potential causes of pelvic discomfort, and a marriage and family therapist to discuss psychosocial factors that may be attributable to sexual dysfunction. She also saw a physical therapist with expertise in pelvic floor rehabilitation, which reduced her pelvic floor pain.

\section{CASE STUDY FOLLOW-UP}

Currently, KS reports she is confident and satisfied with her decision to expand her family despite the challenges associated with PABC treatment.

$\mathrm{KS}$ was followed by the multidisciplinary oncology team for every 3 months the first 2 years and biannually for years 2 through 5 . Despite a 
stressful event such as PABC, KS experienced expert coordinated care. This multidisciplinary and interdisciplinary teamwork was crucial to the successful outcomes for KS and her subsequent delivery of a full-term healthy baby boy.

\section{FUTURE PREGNANCY}

For patients with $\mathrm{PABC}$, the chance of subsequent pregnancy is nearly $70 \%$ lower when compared with the general population, mostly due to treatments with gonadotropic chemotherapy and prolonged treatment periods with tamoxifen for hormone receptor-positive breast disease (Shandley et al., 2017). The rate of return of menses is higher for patients under 40 years of age and for those who received taxane-based chemotherapy (Loibl et al., 2015).

A multidisciplinary approach prior to conception is recommended. Anecdotal evidence suggests a 2-year wait to conceive after treatment and a 5-year wait for those who experienced recurrent stage 1 to 2 disease. Patients with metastatic disease are advised against pregnancy due to limited life expectancy and possible compromise (De Simone \& Pagani, 2017; Pagani et al., 2015). Tamoxifen should be stopped 3 months prior to conception efforts.

\section{CONCLUSIONS}

The prognosis of $\mathrm{PABC}$ is similar to that of breast cancer in the nonpregnant state. Generally, 5-year overall survival is $78 \%$ for the pregnant patient and $81 \%$ for the nonpregnant patient (Amant et al., 2013; Sanchez et al., 2014). Treatment should begin after the diagnosis is established. Surgery options are expanding, and chemotherapy after the first trimester is considered safe. If it is the mother's desire, the aim should be to carry the fetus to term whenever possible. In general, terminating a pregnancy or delaying postpartum treatment does not improve survival (Rovera et al., 2013; Toesca et al., 2014). Future pregnancy is generally not contraindicated (Yu et al., 2017). A pregnancy following breast cancer does not have a negative impact on prognosis (Knabben \& Mueller, 2017).

The management of PABC requires a careful collaborative effort of a multidisciplinary team of providers to offer the most up-to-date options and treatments in PABC (Rovera et al., 2013; Toesca et al., 2014). Advanced practice providers (APPs) are an integral part of a multidisciplinary care team. The APP's role may include evaluation of new patients with breast cancer alongside physician colleagues. Advanced practice providers may order appropriate diagnostic tests, arrange biopsies, and educate patients and families on treatment plans. Additionally, breast cancer survivors are seen by APPs during routine oncology survivorship visits, which may aid in the detection of recurrent disease and include early evaluation, treatment, and prevention of late-term treatment effects.

\section{Acknowledgment}

I would like to thank Beverly Murphy, library specialist at Duke University, for her gracious assistance with literature searches, EndNote reference use, and generous encouragement during this process.

\section{Disclosure}

The author has no conflicts of interest to disclose.

\section{References}

Amant, F., von Minckwitz G., Han, S. N., Bontenbal, M., Ring, A. E., Giermek, J.,...Loibl, S. (2013). Prognosis of women with primary breast cancer diagnosed during pregnancy: Results from an international collaborative study. Journal of Clinical Oncology, 31(20), 2532-2539. https://doi. org/10.1200/JCO.2012.45.6335

Basta, P., Bak, A., \& Roszkowski, K. (2015). Cancer treatment in pregnant women. Contemporary Oncology, 19(5), 354360. https://doi.org/10.5114/wo.2014.46236

Black, K. Z., Nichols, H. B., Eng, E., \& Rowley, D. L. (2017). Prevalence of preterm, low birthweight, and small for gestational age delivery after breast cancer diagnosis: A population-based study. Breast Cancer Research, 19(1), 11. https://doi.org/10.1186/s13058-017-0803-z

Botha, M. H., Rajaram, S., \& Karunaratne, K. (2018). Cancer in pregnancy. International Journal of Gynecology and $\mathrm{Ob}$ stetrics, 143(suppl 2), 137-142. https://doi.org/10.1002/ ijgo.12621

Brooks, J. D., Boice, J. D., Jr., Stovall, M., Reiner, A. S., Bernstein, L., John, E. M.,...Shore, R. E. (2012). Reproductive status at first diagnosis influences risk of radiationinduced second primary contralateral breast cancer in the WECARE study. International Journal of Radiation Oncology, Biology, Physics, 84(4), 917-924. https://doi. org/10.1016/j.ijrobp.2012.01.047

Cordeiro, C. N., \& Gemignani, M. L. (2017). Breast cancer in pregnancy: Avoiding fetal harm when maternal treatment is necessary. Breast Journal, 23(2), 200-205. https://doi.org/10.1111/tbj.12780

Danet, C., Araujo, M., Bos-Thompson, M. A., Portolan, G., Gautier, S., Vanlemmens, L.,...Lacroix, I. (2018). Pregnancy outcomes in women exposed to cancer chemo- 
therapy. Pharmacoepidemiology and Drug Safety, 27(12), 1302-1308. https://doi.org/10.1002/pds.4689

De Simone, V., \& Pagani, O. (2017). Pregnancy after breast cancer: Hope after the storm. Minerva Ginecologica, 69(6), 597-607. https://doi.org/10.23736/s0026-4784.17.04113-2

Framarino-Dei-Malatesta, M., Sammartino, P., \& Napoli, A. (2017). Does anthracycline-based chemotherapy in pregnant women with cancer offer safe cardiac and neurodevelopmental outcomes for the developing fetus? BMC Cancer, 17(1), 777. https://doi.org/10.1186/s12885-0173772-9

Gajjar, K., Martin-Hirsch, P. L., \& Martin, F. L. (2012). Treatment of breast cancer during pregnancy. Lancet Oncology, 13(11), e460. https://doi.org/10.1016/s14702045(12)70417-5

Garrido-Marín, M., Argacha, P. M., Fernández, L., Molfino, F., Martínez-Soler, F., Tortosa, A., \& Gimenez-Bonafé, P. (2019). Full-term pregnancy in breast cancer survivor with fertility preservation: A case report and review of literature. World Journal of Clinical Cases, 7(1), 58-68. https://doi.org/10.12998/wjcc.v7.i1.58

Johansson, A. L. V., Weibull, C. E., Fredriksson, I., \& Lambe, M. (2019). Diagnostic pathways and management in women with pregnancy-associated breast cancer (PABC): No evidence of treatment delays following a first healthcare contact. Breast Cancer Research and Treatment, 174(2), 489-503. https://doi.org/10.1007/s10549-018-05083-x

Johnson, R. H., Anders, C. K., Litton, J. K., Ruddy, K. J., \& Bleyer, A. (2018). Breast cancer in adolescents and young adults. Pediatric Blood \& Cancer, 65(12), e27397. https:// doi.org/10.1002/pbc.27397

Knabben, L., \& Mueller, M. D. (2017). Breast cancer and pregnancy. Hormone Molecular Biology and Clinical Investigation, 32(1). https://doi.org/10.1515/hmbci-2017-0026

Lambertini, M., Kamal, N. S., Peccatori, F. A., Del Mastro, L., \& Azim, H. A., Jr. (2015). Exploring the safety of chemotherapy for treating breast cancer during pregnancy. $E x$ pert Opinion on Drug Safety, 14(9), 1395-1408. https://doi. org/10.1517/14740338.2015.1061500

Loibl, S., Schmidt, A., Gentilini, O., Kaufman, B., Kuhl, C., Denkert, C.,...Amant, F. (2015). Breast cancer diagnosed during pregnancy: Adapting recent advances in breast cancer care for pregnant patients. JAMA Oncology, 1(8), 1145-1153. https://doi.org/10.1001/jamaoncol.2015.2413

National Comprehensive Cancer Network. (2018). NCCN Clinical Practice Guidelines in Oncology: Breast cancer. V1.2018. Retrieved from https://www.nccn.org/professionals/physician_gls/pdf/breast.pdf

Pagani, O., Ruggeri, M., Manunta, S., Saunders, C., Peccatori, F., Cardoso, F.,...Partridge, A. H. (2015). Pregnancy after breast cancer: Are young patients willing to participate in clinical studies? Breast, 24(3), 201-207. https://doi. org/10.1016/j.breast.2015.01.005
Pistilli, B., Bellettini, G., Giovannetti, E., Codacci-Pisanelli, G., Azim, H. A., Jr., Benedetti, G.,...Peccatori, F. A. (2013). Chemotherapy, targeted agents, antiemetics and growthfactors in human milk: How should we counsel cancer patients about breastfeeding? Cancer Treatment Reviews, 39(3), 207-211. https://doi.org/10.1016/j.ctrv.2012.10.002

Rovera, F., Chiappa, C., Coglitore, A., Baratelli, G. M., Fachinetti, A., Marelli, M.,...Dionigi, R. (2013). Management of breast cancer during pregnancy. International Journal of Surgery (London, England), 11(suppl 1), S64-S68. https:// doi.org/10.1016/s1743-9191(13)60020-5

Sanchez, C., Acevedo, F., Medina, L., Ibanez, C., Razmilic, D., Elena Navarro, M., \& Camus, M. (2014). Breast cancer and pregnancy: A comparative analysis of a Chilean cohort. Ecancermedicalscience, 8, 434. https://doi. org/10.3332/ecancer.2014.434

Shachar, S. S., Gallagher, K., McGuire, K., Zagar, T. M., Faso, A., Muss, H. B.,...Anders, C. K. (2017). Multidisciplinary management of breast cancer during pregnancy. Oncologist, 22(3), 324-334. https://doi.org/10.1634/theoncologist.2016-0208

Shandley, L. M., Spencer, J. B., Fothergill, A., Mertens, A. C., Manatunga, A., Paplomata, E., \& Howards, P. P. (2017). Impact of tamoxifen therapy on fertility in breast cancer survivors. Fertility and Sterility, 107(1), 243-252.e245. https://doi.org/10.1016/j.fertnstert.2016.10.020

Shlensky, V., Hallmeyer, S., Juarez, L., \& Parilla, B. V. (2017). Management of breast cancer during pregnancy: Are we compliant with current guidelines? AJP Reports, 7(1), e39-e43. https://doi.org/10.1055/s-0037-1599133

Toesca, A., Gentilini, O., Peccatori, F., Azim, H. A., Jr., \& Amant, F. (2014). Locoregional treatment of breast cancer during pregnancy. Gynecological Surgery, 11(4), 279284. https://doi.org/10.1007/s10397-014-0860-6

Tomasi-Cont, N., Lambertini, M., Hulsbosch, S., Peccatori, A. F., \& Amant, F. (2014). Strategies for fertility preservation in young early breast cancer patients. Breast, 23(5), 503-510. https://doi.org/10.1016/j.breast.2014.05.024

Trivers, K. F., Fink, A. K., Partridge, A. H., Oktay, K., Ginsburg, E. S., Li, C., \& Pollack, L. A. (2014). Estimates of young breast cancer survivors at risk for infertility in the U.S. Oncologist, 19(8), 814-822. https://doi.org/10.1634/theoncologist.2014-0016

Yu, H. H., Cheung, P. S., Leung, R. C., Leung, T. N., \& Kwan, W. H. (2017). Current management of pregnancy-associated breast cancer. Hong Kong Medical Journal, 23(4), 387-394. https://doi.org/10.12809/hkmj166049

Zagouri, F., Sergentanis, T. N., Chrysikos, D., Dimitrakakis, C., Tsigginou, A., Zografos, C. G.,...Papadimitriou, C. A. (2013). Taxanes for breast cancer during pregnancy: A systematic review. Clinical Breast Cancer, 13(1), 16-23. https://doi.org/10.1016/j.clbc.2012.09.014 